\title{
BMJ Open Mapping the study topics and characteristics of HIV pre-exposure prophylaxis research literature: a protocol for a scoping review
}

\author{
Emiko Kamitani, ${ }^{1}$ Adebukola H Johnson, ${ }^{1,2}$ Megan Wichser, ${ }^{1,2}$ Yuko Mizuno, ${ }^{1}$ \\ Julia B DeLuca, ${ }^{1}$ Darrel H Higa ${ }^{1}$
}

To cite: Kamitani $\mathrm{E}$, Johnson AH, Wichser M, et al. Mapping the study topics and characteristics of HIV pre-exposure prophylaxis research literature: a protocol for a scoping review. BMJ Open 2019;9:e024212. doi:10.1136/ bmjopen-2018-024212

- Prepublication history and additional material for this paper are available online. To view these files, please visit the journal online (http://dx.doi. org/10.1136/bmjopen-2018024212).

Received 15 May 2018 Revised 14 December 2018 Accepted 9 April 2019

\section{Check for updates}

(c) Author(s) (or their employer(s)) 2019. Re-use permitted under CC BY-NC. No commercial re-use. See rights and permissions. Published by BMJ.

${ }^{1}$ Division of HIV/AIDS Prevention, Centers for Disease Control and Prevention Prevention Research Centers, Atlanta, Georgia, USA ${ }^{2}$ ICF International Inc, Fairfax, Virginia, USA

Correspondence to Dr Emiko Kamitani; ybo9@cdc.gov

\section{ABSTRACT}

Introduction The research literature addressing preexposure prophylaxis (PrEP) has increased considerably over the last decade. To better understand the research areas and explore research gaps, we will conduct a scoping review to map study topics and describe study characteristics and populations in publications focused on PrEP. The purpose of this protocol is to describe planned methods for the scoping review.

Methods and analysis We will implement a comprehensive systematic literature search to identify PrEP citations in the United States Centres for Disease Control and Prevention HIV/AIDS Prevention Research Synthesis Project database that is unique and extensively focuses on HIV/sexually transmitted infections/hepatitis. We will screen and include studies that are (1) focused on HIV PrEP, (2) primary research with human participants and (3) published in English. Two reviewers will independently abstract data on authors' names, study years, countries, population characteristics and design. To describe and summarise study topics, we will use 19 codes and five categories that were developed from a preliminary study. The five categories are category 1: potential PrEP user/ prescriber (behaviours/issues for potential PrEP takers/ healthcare professionals), category 2: considerations while on PrEP (experiences of and problems related to staying on or prescribing PrEP), category 3: PrEP efficacy and safety (biomedical aspects and medication efficacy), category 4: methods of and experiences with PrEP clinical trials (possesses/experiences of clinical trials) and category 5: cost-effectiveness or economic evaluation (cost studies). Data will be analysed with descriptive statistics.

Ethics and dissemination The findings will be presented at HIV-related conferences and published in peer-review journals.

\section{INTRODUCTION}

Approximately 36.7 million people are living with HIV globally; about 1.8 million are infected with HIV every year. ${ }^{1}$ The Joint United Nations Programme on HIV/AIDS released the HIV strategic plan, 'Getting to Zero, ${ }^{2}$ to achieve the United Nations Millennium Development Goals. ${ }^{3}$ One of their
Strengths and limitations of this study

- Our study will describe the totality of evidence regarding pre-exposure prophylaxis (PrEP) studies in terms of design and characteristics of the studies currently published in the literature.

- Our scoping review will map the PrEP research literature by identifying knowledge gaps and understudied populations in the PrEP research literature.

- Our search is limited to studies published in English only, and our review cannot rule out publication bias.

goals is to achieve zero new HIV infections by cutting the number of sexual transmission events in half, eliminating vertical transmission and preventing HIV transmission among substance users. ${ }^{2}$

Pre-exposure prophylaxis (PrEP) plays an important role in achieving the goal of zero new HIV infections. The daily oral HIV PrEP pill, emtricitabine/tenofovir (Truvada), is known to reduce HIV acquisition in clinical trials as well as community-based ('realworld') studies. ${ }^{4-7}$ In 2012, the USA was the first country to approve Truvada for use as PrEP and the WHO released the first PrEP clinical practice guideline. ${ }^{8}$

Following PrEP's approval and introduction of the WHO guideline, the PrEP-related research literature grew significantly. As the effectiveness of PrEP was established, behavioural and structural factors associated with PrEP are becoming important to understand. For example, PrEP effectiveness is strongly related to behavioural factors such as medication adherence. ${ }^{4}$ Persons using PrEP may be engaging in more condomless sex and other high-risk behaviours compared with non-PrEP users. ${ }^{9}$ In addition, new administration and dosage schedules for PrEP have been introduced; these include other types of drugs (eg, tenofovir only, cabotegravir), dose 
(eg, on demand, four times a week) and routes (eg, topical gel, injectable).$^{10-12}$ These new ideas have initiated other areas for research and evaluation, and the PrEP literature has grown considerably over the last decade.

To better understand this emerging research area and explore research gaps, we will conduct a scoping review of the PrEP research literature. The objective of a scoping review is to identify the most common topics studied and evidence gaps in the literature. ${ }^{13}$ To our knowledge, no scoping review on the PrEP research literature has been published to date. In this protocol, we will provide information on the objectives of the scoping review, search strategy details, study selection and inclusion, and plans for data abstraction and analysis. We followed the Preferred Reporting Items for Systematic Reviews and Meta-Analyses Protocols checklist for reporting (see online supplementary appendix 1). ${ }^{14}$

\section{STUDY OBJECTIVES}

The purpose of our scoping review is to explore the type and extent of the research literature available on PrEP. The specific objectives are to:

- Identify and map the most common topic areas.

- Classify study characteristics (study years, countries, population characteristics and designs).

- Identify research gaps.

\section{METHODS AND ANALYSIS \\ Methodology}

We will use the scoping review methodological framework introduced by Arksey and O'Malley ${ }^{13}$ and the Preferred Reporting Items for Systematic Reviews and Meta-Analyses extension for Scoping Reviews statement checklist as a guideline to structure the scoping review. ${ }^{15}$ Scoping review methodology is similar to that of systematic reviews; thus, we will apply established systematic review techniques to locate, screen, assess and abstract data to identify topic areas and research gaps.

\section{Search strategy}

We developed a systematic literature search to identify PrEP-related citations. A subject matter expert identified 24 'gold standard' citations in the PrEP literature. The expert was the United States Centres for Disease Control and Prevention (CDC) physician epidemiologist who is the lead author of the CDC PrEP clinical guideline and has extensively published on PrEP.

A librarian examined these citations to identify possible PrEP keywords and phrases. Search terms were tested to pinpoint essential keywords and Medial Subject Headings (MeSH; indexing) terms. The strategy captures citations on infectious diseases (HIV, sexually transmitted infections (STI) or hepatitis C) cross-referenced against PrEP terms (pre-exposure prophylaxis, chemoprophylaxis) published since 2000 with no language restriction. The full search was first developed in MEDLINE (OVID). Once finalised, the MEDLINE search was tailored to other database's unique indexing in EMBASE (OVID), PsycINFO (OVID) and CINAHL (EBSCOhost) (see online supplementary appendix 2).

All citations retrieved with the searches were uploaded to the CDC HIV/AIDS Prevention Research Synthesis (PRS) Project database. This unique comprehensive database includes citations related to HIV, AIDS and STI prevention. We conduct annual electronic and manual searching of numerous databases to upload newly published studies on these topics. The process of creating a comprehensive systematic literature search strategy for the PRS database has been published elsewhere. ${ }^{16}$ As of November 2018, >92000 related citations were included in the PRS database.

\section{Identifying relevant studies}

We will search the PRS database for PrEP-related citations. PRS staff will identify HIV PrEP citations by reviewing titles and abstracts. All identified citations will be exported to the systematic review software DistillerSR (Evidence Partners, Ottawa, Canada) for data management, citation screening and data abstraction.

Inclusion criteria for this review are primary studies: (1) focusing on PrEP to prevent HIV, (2) using human subjects and (3) published in English. We will exclude systematic reviews and literature reviews, commentaries, guidelines, protocols, letters to editors, laboratory (eg, in vitro) or preclinical studies (eg, animal studies), grey literature (eg, newsletters) and conference abstracts. Research studies estimating drug efficacy and/or drug resistance for people living with HIV as well as studies focused on PrEP to prevent diseases other than HIV (eg, other STI) will be also excluded.

A three-step approach will be used to identify eligible studies. First, one reviewer will screen the citations by title and abstract to identify those that are related to PrEP and published in English. Citations that are excluded by the reviewer will be verified by a second reviewer. Second, two reviewers will independently review the full text of the included citation to determine whether the primary study was conducted with humans. We will also exclude ineligible studies (eg, systematic reviews) at this level. Disagreements will be resolved through discussion. Finally, if there is a lack of agreement between the two reviewers, a third reviewer will resolve the discrepancy. All forms will be pilot tested and revised as necessary.

\section{Data abstraction}

For eligible citations, two reviewers will independently abstract data on author names, publication year, countries, population characteristics, design and topics. We will abstract study population characteristics using the primary study's inclusion criteria and identify study topics by reviewing the stated purposes or objectives. When the pair of reviewers fail to reach agreement, a third reviewer will resolve the discrepancy. 


\section{Mapping study topics}

To map study topics, we developed preliminary codes and categories (table 1). First, we generated initial codes to describe the study topics via literature reviews and consultations with the subject matter experts. These experts included the CDC physician epidemiologist who helped us develop the search strategy and another CDC behavioural scientist with PrEP expertise evidenced in numerous publications. A code succinctly describes or represents a phenomenon or concept. Second, we assigned at least one code to each citation from a sample of 195 PrEP-related citations that were identified via a search in the PRS database. Third, by using a thematic analysis technique, ${ }^{17}$ we sorted and grouped these codes into larger concept areas by reviewing the relationships to other codes and assigned a broader 'category'. Codes and categories are not mutually exclusive. In this preliminary study, we identified 19 codes that were collapsed into five categories: (1) potential PrEP user/prescriber, (2) considerations while on PrEP, (3) PrEP efficacy and safety, (4) methods of and experiences with PrEP clinical trials and (5) cost-effectiveness or economic evaluation. The following describes each of the categories with specific examples.

\section{Category 1: potential PrEP user/prescriber}

This category will capture studies discussing behavioural aspects or criteria for potential PrEP users or providers who may be considering prescribing PrEP, but have not actually done so. Studies assigned in this category include those that report barriers and facilitators for taking or prescribing PrEP. This category consists of four codes described below.

We will assign the code access/routinehealthcare visit to studies discussing barriers to access PrEP prescriptions or lack of feasibility of PrEP programmes due to structural issues (eg, costs to the patient, lack of providers who can prescribe PrEP or inability of healthcare providers to prescribe PrEP at point of care). Studies on other types of barriers to take PrEP (eg, barriers related to cognition and emotion such as stigma and denial) will be coded as acceptability/willingness. We will assign the code knowledge/awareness to studies assessing visibility of PrEP and PrEP candidacy/HIVrisk to studies discussing the association between PrEP uptake and risk perceptions, frequency of HIV testing, risky behaviours and number of sex partners.

\section{Category 2: considerations while on PrEP}

This category will capture studies that report experiences of and issues/problems related to starting or staying on PrEP encountered by PrEP users or prescribers. This category consists of nine codes.

We will assign codes adherence to studies assessing adherence to PrEP and retention and re-engagement in care to studies with some aspect of retention/re-engagement in PrEP care. The code adverse event will be assigned to studies discussing adverse events as a barrier to taking PrEP and risk compensation will be assigned to studies assessing the frequency of HIV risk behaviours (eg, condomless sex, injection drug use) or reporting the incidence of STI while on PrEP. Studies assessing subjective judgments and beliefs about HIV risk while on PrEP will be coded as risk perception. The code conception will be assigned to studies reporting PrEP use among HIV-serodiscordant couples desiring a child. This category also includes studies that assess disclosure of PrEP use to partners, PrEP user characteristics and the experiences of taking PrEP other than adherence and adverse events, which will be assigned the code PrEP user issues and characteristics/PrEPuptake. Experiences of prescribing PrEP among healthcare providers and/or managing a PrEP clinic will be assigned the code PrEP prescription/PrEP clinic. Other study topics in this category include the type and effect on PrEP uptake of routine HIV testing and screening for other health issues, such as mental health disorders. These studies will be assigned the code routine HIV testing/health screening.

\section{Category 3: PrEP efficacy and safety}

This category will capture studies that focus on biomedical aspects of PrEP and medication efficacy, including new modes of PrEP medications (eg, type, dose and route of administration). There are two codes in this category.

We will assign the code effectiveness/safety/drug resistance to studies assessing medication efficacy (eg, reduction of HIV seroconversion), harms resulting from the use of medication, and sensitivity to other antiretroviral medications. Examples of studies with this code include randomised controlled trials to test PrEP efficacy as well as studies monitoring HIV disease progression among people who seroconverted while on PrEP. We will also assign the code to studies assessing the biomedical aspects of contraceptive drugs among women on PrEP (eg, drug safety or interactions). For studies simulating the impact of PrEP on HIV incidence, and drug effectiveness or resistance, we will assign the code Estimate impact and effectiveness/drug resistance. We anticipate that studies with this code will usually be mathematical modelling studies.

\section{Category 4: methods of and experiences with PrEP clinical trials}

This category will capture studies focusing on processes or experiences of clinical trials and contains two codes.

We will assign the code Trial methods and characteristics to PrEP study protocols that explain methods used in a clinical trial or intervention, or those that discuss ethical issues of conducting PrEP trials. Another example includes studies that only report baseline data. We will assign the code Trial experiences to studies that report trial participants' experiences (eg, stigma experienced during their participation and their partners' reactions).

\section{Category 5: cost-effectiveness or economic evaluation}

This category has two codes, Cost-effectiveness and Economic evaluation, and will capture all types of cost studies. The code Cost-effectiveness will be assigned to studies comparing the cost of PrEP treatment and HIV testing, and to those focusing on prompt treatment. These reports are usually 
Table 1 Mapping study topics: categories and codes with examples of study topics for each

\section{Categories(n=5)}

1. Potential PrEP user/prescriber

Kr

Knowledge/awareness

Access/routine healthcare visit (structural)

PrEP candidacy/HIV risk

2. Consideration while on PrEP

\section{Adherence}

Retention/re-engagement in care

Risk compensation

Risk perception

Adverse event

PrEP user issues and characteristics/

PrEP uptake

PrEP prescription/PrEP clinic

Conception (not with birth control pill)

Routine HIV testing/health screening

\section{PrEP efficacy and safety}

Effectiveness/safety/drug resistance with PrEP clinical trials

Estimate impact and effectiveness/drug resistance

Trial' method/characteristics

\section{Examples of study topics}

Barriers/facilitators/stigma to take PrEP PrEP willingness

Feasibility of starting PrEP or programme

Awareness of PrEP

Knowledge of PrEP

Routine HIV testing

Men's healthcare

Feasibility of PrEP clinic

Risk perceptions

HIV testing/status

Risk factors/behaviours

Who benefits the most from PrEP

Number of sex partners

PrEP eligibility

\section{Adherence}

Adherence measurement

Retention in care

Re-engagement in care

Condomless sex while on PrEP

Injection drug uses

Incidence of STIs

HIV risk perception while on PrEP

Adverse events

PrEP uptake/use

Disclose to partner, stigma

PrEP user's experience/characteristics

PrEP prescribe (for provider)

PrEP clinic characteristics

PrEP interaction with hormone

HIV serodiscordant couples desiring a child

Incidence of pregnancy

Type and effect of HIV testing

Screening for mental health problems

Drug resistance

HIV disease progression

Effectiveness of PrEP

PrEP safety

Estimate impact of PrEP

Estimate effectiveness of PrEP

Estimate drug resistance

Study participants' characteristics

Study method

Study participants' experience

Experience of researchers

Influence of participants' partner 
Table 1 Continued

\begin{tabular}{lll} 
Categories $(\mathbf{n}=5)$ & Codes $(\mathbf{n}=19)$ & Examples of study topics \\
$\begin{array}{l}\text { 5. Cost-effectiveness or economic } \\
\text { evaluation }\end{array}$ & Cost-effectiveness & Cost-effectiveness \\
& Economic evaluation & Cost unity/benefit/minimisation \\
\hline
\end{tabular}

PrEP, pre-exposure prophylaxis; STIs, sexually transmitted infections.

mathematical modelling studies. Example of studies with the code Economic evaluation include studies that discuss cost utility, cost benefit, and cost minimization.

These codes and categories are considered preliminary. We will confirm and modify them as needed by using constant comparison methods that allow for continuous validation with subsequent citations through the scoping review process. ${ }^{18}$

\section{Data analysis}

We will summarise the codes and categories for study topics using descriptive statistics (eg, frequencies, percentages). Publication year, country where the study was conducted, population characteristics (eg, age, race, gender, sexual orientation, risk category), and research design (eg, cross sectional, randomised controlled trials, cohort) will also be analysed using descriptive statistics. We will not synthesise the findings from the included studies since our goal is to provide a scope or broad perspective of the research literature. ${ }^{13}$

\section{Patient and public involvement}

No patients and public were involved to develop this protocol.

\section{ETHICS AND DISSEMINATION}

This review does not require ethics approval since we are using previously published data. We will present our findings at HIV-related conferences and publish in a peer-reviewed journal.

\section{LIMITATIONS}

One limitation of our scoping review is the exclusion of non-English or grey literature. By excluding these literatures, we cannot rule out publication bias in our findings since we may be missing studies from non-English speaking countries and with negative or null findings. We will not assess study quality since it is generally considered to be optional for scoping reviews. ${ }^{19}$ Due to the lag in adding and indexing articles in various online databases, our review may not include the most recent publications and may not represent the latest knowledge about PrEP. Another limitation is that we will use inclusion criteria of the included primary studies to determine study participants' characteristics, and the study's stated purposes or objectives for study topics. Thus our review will not capture other information described or implied elsewhere in the full text (eg, research sites and reported sample characteristics).

\section{DISCUSSION}

This scoping review will contribute to the PrEP field by examining the PrEP literature to map study topics, describe study characteristics and populations and identify research gaps. In particular, the review will identify understudied populations and behavioural research topics related to PrEP that could be the focus of future primary studies. By providing a broad overview of the PrEP literature, we hope that our review will contribute to HIV prevention efforts to achieve zero new HIV infections.

Contributors EK developed the research question and study methods and drafted the protocol. DHH contributed to critical review and revision of the manuscript. $\mathrm{JBD}$ conducted searches for the preliminary study and drafted the search strategy for the protocol. AHA, MW and YM aided in coding the preliminary study and contributed to the drafting and editing. All authors have read and approved the final manuscript.

Disclaimer The findings and conclusions in this report are those of the authors and do not necessarily represent the official position of the United States Centers for Disease Control and Prevention.

Competing interests None declared.

Patient consent for publication Not required.

Provenance and peer review Not commissioned; externally peer reviewed.

Open access This is an open access article distributed in accordance with the Creative Commons Attribution Non Commercial (CC BY-NC 4.0) license, which permits others to distribute, remix, adapt, build upon this work non-commercially, and license their derivative works on different terms, provided the original work is properly cited, appropriate credit is given, any changes made indicated, and the use is non-commercial. See: http://creativecommons.org/licenses/by-nc/4.0/.

\section{REFERENCES}

1. Wold Health Organization. World AIDS Day around the world. Programmes. 2017. http://www.who.int/campaigns/aids-day/2017/ en/ [Accessed 2 Jan 2018].

2. Joint United Nations Programme on HIV/AIDS (UNAIDS). Getting To Zero: 2011-2015 strategy. 2010. http://www.unaids.org/sites/default/ files/sub_landing/files/JC2034_UNAIDS_Strategy_en.pdf [Accessed 22 Dec].

3. Wold Health Organization. Millennium Development Goals (MDGs) Health topics. 2017. http://www.who.int/topics/millennium_ development_goals/about/en/ [Accessed 29 Dec 2017].

4. Grant RM, Lama JR, Anderson PL, et al. Preexposure chemoprophylaxis for HIV prevention in men who have sex with men. N Engl J Med 2010;363:2587-99.

5. Liu AY, Cohen SE, Vittinghoff E, et al. Preexposure Prophylaxis for HIV Infection Integrated With Municipal- and Community-Based Sexual Health Services. JAMA Intern Med 2016;176:75-84.

6. McCormack S, Dunn DT, Desai M, et al. Pre-exposure prophylaxis to prevent the acquisition of HIV-1 infection (PROUD): effectiveness results from the pilot phase of a pragmatic open-label randomised trial. Lancet 2016;387:53-60. 
7. Volk JE, Marcus JL, Phengrasamy T, et al. No New HIV Infections With Increasing Use of HIV Preexposure Prophylaxis in a Clinical Practice Setting. Clin Infect Dis 2015;61:1601-3.

8. U.S. Food and Drug Administration. Truvada for PrEP Fact Sheet: Ensuring Safe and Proper Use. 2012. https://www.fda.gov/ downloads/Drugs/DrugSafety/PostmarketDrugSafetylnformationf orPatientsandProviders/UCM312290.pdf [Accessed Jul].

9. Newcomb ME, Mongrella MC, Weis B, et al. Partner Disclosure of PrEP Use and Undetectable Viral Load on Geosocial Networking Apps: Frequency of Disclosure and Decisions About Condomless Sex. J Acquir Immune Defic Syndr 2016;71:200-6.

10. National Institutes of Health. Safety and Efficacy Study of Injectable Cabotegravir Compared to Daily Oral Tenofovir Disoproxil Fumarate/ Emtricitabine (TDF/FTC), For Pre-Exposure Prophylaxis in HIVUninfected Cisgender Men and Transgender Women Who Have Sex With Men. https://clinicaltrials.gov/ct2/show/NCT02720094 [Accessed 8 May 2018].

11. Molina JM, Capitant C, Spire B, et al. On-Demand Preexposure Prophylaxis in Men at High Risk for HIV-1 Infection. N Engl J Med 2015;373:2237-46.

12. Grant RM, Anderson PL, McMahan V, et al. Uptake of pre-exposure prophylaxis, sexual practices, and HIV incidence in men and transgender women who have sex with men: a cohort study. Lancet Infect Dis 2014;14:820-9.

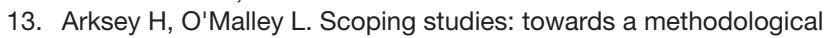
framework. Int J Soc Res Methodol 2005;8:19-32.

14. Shamseer L, Moher D, Clarke M, et al. Preferred reporting items for systematic review and meta-analysis protocols (PRISMA-P) 2015: elaboration and explanation. BMJ 2015;349:g7647.

15. Tricco AC, Lillie E, Zarin W, et al. PRISMA extension for scoping reviews (PRISMA-ScR): Checklist and explanation. Ann Intern Med 2018;169:467.

16. DeLuca JB, Mullins MM, Lyles CM, et al. Developing a comprehensive search strategy for evidence based systematic reviews. Evid Based Libr Inf Pract 2008;3:3.

17. Braun V, Clarke V. Using thematic analysis in psychology. Qual Res Psychol 2006;3:77-101.

18. Glaser BG, Strauss AL. The Discovery of Grounded Theory: Strategies for Qualitative Research. Chicago: Aldine Pub. Co, 1967.

19. Rumrill PD, Fitzgerald SM, Merchant WR. Using scoping literature reviews as a means of understanding and interpreting existing literature. Work 2010;35:399-404. 